\section{Structural and functional analysis of the DOT1L-AF10 complex reveals mechanistic insights into MLL-AF10- associated leukemogenesis}

\author{
Heng Zhang, 1,5 Bo Zhou, ${ }^{2,5}$ Su Qin, ${ }^{1,3,5}$ Jing $\mathrm{Xu}^{2}{ }^{2}$ \\ Rachel Harding, ${ }^{1}$ Wolfram Tempel, ${ }^{1}$ Vinod Nayak, ${ }^{1}$ \\ Yanjun Li, ${ }^{1}$ Peter Loppnau, ${ }^{1}$ Yali Dou, ${ }^{2}$ \\ and Jinrong $\mathrm{Min}^{1,4}$
}

\begin{abstract}
${ }^{1}$ Structural Genomics Consortium, University of Toronto, Toronto, Ontario M5G 1L7, Canada; ${ }^{2}$ Department of Pathology, University of Michigan, Ann Arbor, Michigan 48109, USA; ${ }^{3}$ Life Science Research Center, Southern University of Science and Technology, Shenzhen 518055, China; ${ }^{4}$ Department of Physiology, University of Toronto, Toronto, Ontario M5G 1L7, Canada
\end{abstract}

The mixed-lineage leukemia (MLL)-AF10 fusion oncoprotein recruits DOT1L to the homeobox A (HOXA) gene cluster through its octapeptide motif leucine zipper (OM-LZ), thereby inducing and maintaining the MLLAF10-associated leukemogenesis. However, the recognition mechanism between DOT1L and MLL-AF10 is unclear. Here, we present the crystal structures of both apo $\mathrm{AF}^{\mathrm{OM}-\mathrm{LZ}}$ and its complex with the coiled-coil domain of DOT1L. Disruption of the DOT1L-AF10 interface abrogates MLL-AF10-associated leukemic transformation. We further show that zinc stabilizes the DOT1L-AF10 complex and may be involved in the regulation of the HOXA gene expression. Our studies may also pave the way for the rational design of therapeutic drugs against $M L L$-rearranged leukemia.

Supplemental material is available for this article.

Received January 11, 2018; revised version accepted March 1, 2018.

Aberration of the mixed-lineage leukemia $(M L L$; also known as $M L L 1, A L L 1, H R X$, or $H T R X)$ gene is widely implicated in pathogenesis of acute leukemia (Ziemin-van der Poel et al. 1991). One major MLL aberration is the $M L L$ rearrangement, which is found in $\sim 10 \%$ of acute myeloid leukemia (AML) and acute lymphoblastic leukemia (ALL) cases with a relatively dismal prognosis (Muntean and Hess 2012). The $M L L$ gene encodes a histone H3 Lys4 (H3K4) methyltransferase that positively regulates homeobox (HOX) gene expression (Benjamin et al. 1995). Several functional domains involved in chromatin association (CXXC, AT hooks, plant homeodomain fingers, and bromodomain) are present in the $\mathrm{N}$-terminal portion (Cos-

[Keywords: DOT1L; MLL-AF10; crystal structure; leukemogenesis] ${ }^{5}$ These authors contributed equally to this work. Corresponding author: jr.min@utoronto.ca

Article published online ahead of print. Article and publication date are online at http://www.genesdev.org/cgi/doi/10.1101/gad.311639.118. grove and Patel 2010), while within the C-terminal portion lies a SET [Su(var)3-9, enhancer of zeste, and trithorax] domain that catalyzes H3K4 methylation, an epigenetic mark of active transcription (Shilatifard 2012). Chromosomal rearrangements generate MLL fusion proteins in which the N-terminal portion of MLL is fused to one of $\sim 70$ distinct partner proteins (Slany 2016). The N-terminal chromatin-binding domains (AT hooks and CXXC) are consistently retained in the MLL fusion proteins, whereas the SET domain is absent. Intriguingly, most frequent translocation partners implicated in leukemia are transcriptional activators, which is in agreement with the notion that the target gene activation of MLL is the fundamental mechanism in $M L L$-rearranged leukemia.

The MLL-AF10 fusion is widely distributed among AML subtypes (DiMartino et al. 2002; Meyer et al. 2006). AF10 together with AF4, AF6, AF9, and ENL are the most common fusion partners, found in $>80 \%$ of all $M L L$-rearranged leukemia (Krivtsov and Armstrong 2007; Thirman 2017). The MLL-AF10 fusion protein retains the octapeptide motif (OM) leucine zipper (LZ) region of AF10 (AF10 ${ }^{\mathrm{OM}-\mathrm{LZ}}$ ), which recruits the $\mathrm{H} 3 \mathrm{~K} 79$ methyltransferase DOT1L, resulting in H3K79 hypermethylation and dysregulation of HOXA and MEIS1 expression (Okada et al. 2005). Previous studies have demonstrated that $M L L$-rearranged leukemia depends on aberrant H3K79 methylation and up-regulation of $H O X A$ gene clusters (Ayton and Cleary 2003; Okada et al. 2005; Chen et al. 2013). The OM-LZ domain of AF10 is essential for both MLL-AF10- and CALM-AF10-induced leukemogenesis (DiMartino et al. 2002; Okada et al. 2006). AF10 ${ }^{\mathrm{M}-\mathrm{LZ}}$ is also known to interact with the coiled-coil domains of GAS41 and Ikaros (Okada et al. 2006; Yoshida et al. 2006), suggesting a more general scaffolding role for the OM-LZ domain of AF10. AF10 has been identified as a key regulator of progressive H3K79 methylation and HOXA gene expression in various AML subtypes (Deshpande et al. 2014), indicating that disruption of DOT1L-AF10 association would be an attractive therapeutic option for a wide spectrum of AML. However, the molecular mechanism of how AF10 recognizes DOT1L in $M L L$-rearranged leukemia remains unknown.

Here we identified and characterized the coiled-coil domain of DOT1L as the AF10-binding site by biophysical and structural analyses. Our in vivo results revealed that disruption of the DOT1L-AF10 binding impairs the transforming ability of MLL-AF10 and decreases the HOXA gene expression. Our crystal structures also revealed a previously uncharacterized zinc-binding site in the DOT1LAF10 complex. Zinc stabilizes the complex formation and might contribute to the regulation of Hoxa10 gene expression. In addition to elucidating the DOT1L-AF10 recognition mechanisms, our results also shed light on the potential development of DOT1L-AF10-specific therapeutic drugs in AML.

(C) 2018 Zhang et al. This article is distributed exclusively by Cold Spring Harbor Laboratory Press for the first six months after the full-issue publication date (see http://genesdev.cshlp.org/site/misc/terms.xhtml). After six months, it is available under a Creative Commons License (Attribution-NonCommercial 4.0 International), as described at http://creativecommons.org/licenses/by-nc/4.0/. 


\section{Results and Discussion}

\section{AF10 binds to the coiled-coil domain of DOT1L}

The OM-LZ region of AF10 (AF10 ${ }^{\mathrm{OM}-\mathrm{LZ}}$ ) has been reported previously to bind to DOT1L (Okada et al. 2005). As the central region of the DOT1L is predicted to fold into three coiled-coil domains (Fig. 1A; Supplemental Fig. S1A,B; Finn et al. 2011), we hypothesized that the coiled-coil interactions between the OM-LZ region of AF10 and the coiled-coil domains of DOT1L might mediate the DOT1L-AF10 binding. Our GST pull-down assays using recombinant proteins indeed revealed that the coiledcoil domains of DOT1L bound to AF10 ${ }^{\mathrm{M}-\mathrm{LZ}}$ (Fig. 1B). To further map the AF10-binding region on DOT1L, we produced recombinant proteins for each individual coiled-coil domain of DOT1L. Our isothermal titration calorimetry (ITC) studies showed that the first $\left(\mathrm{DOT} 1 \mathrm{~L}^{\mathrm{CC} 1}\right.$ ) and the second (DOT1L ${ }^{\mathrm{CC} 2}$ ) domains exhibit submicromolar affinities toward AF10. In addition, the third coiled-coil domain (DOT1L ${ }^{\mathrm{CC} 3}$ ) binds AF10 with micromolar affinity (Fig. 1C). Thus, DOT1L engages the AF10 binding at three sites. Both the coiled-coil domains of DOT1L and the OM-LZ region of AF10 are evolutionarily conserved across species (Supplemental Figs. S1A, 2A), suggesting a conserved binding mode between DOT1L and AF10. Sequence alignment shows that AF10 and AF17 share a conserved C-terminal OM-LZ domain (Supplemental Fig. S2A), suggesting that AF17 might bind to DOT1L in a manner similar to that of AF10. In-

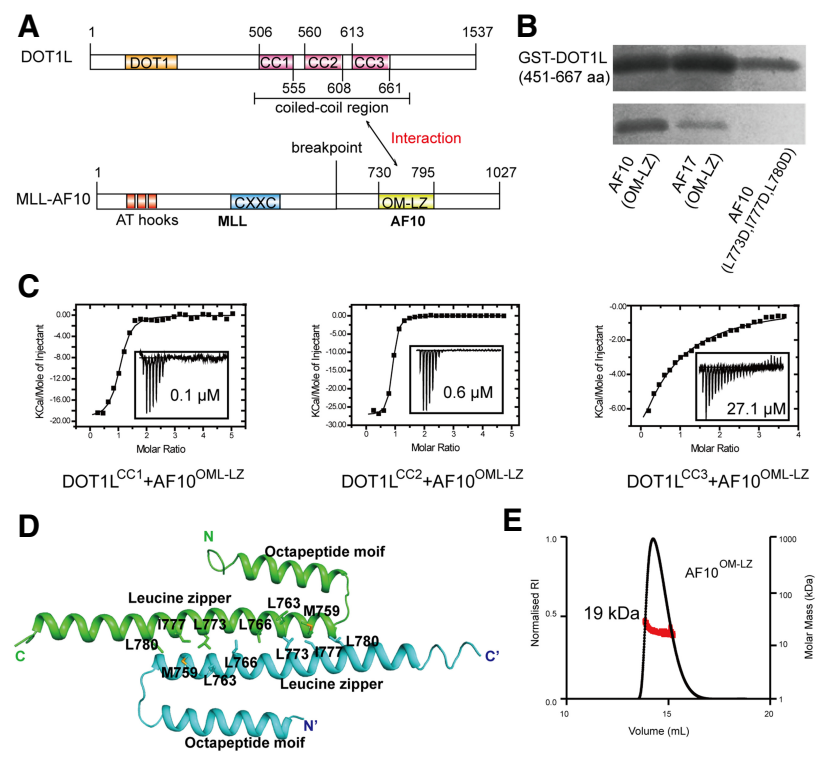

Figure 1. The coiled-coil region of DOT1L binds to AF10 and AF17. (A) Schematic diagrams of the domain organization of DOT1L and MLL-AF10. Interactions between DOT1L and AF10 are indicated by a two-way arrow. There are three coiled-coil regions in DOT1L. (B) The coiled-coil region of DOT1L robustly binds to AF10 and AF17 by GST pull-down assay. Triple mutation AF10 $1773 \mathrm{D}$, I777D, L780D abolishes the DOT1L binding. $(C)$ ITC-based measurements quantifying the binding affinities between the coiled-coil region of DOT1L and AF10 10 -LZ . (NB) No binding. (D) Cartoon representation of the AF10 ${ }^{\mathrm{OM}-\mathrm{LZ}}$ structure. The two chains are colored cyan and green, respectively. The residues involved in the dimer interface are shown in stick representations. $(E)$ Size exclusion chromatography coupled with multiangle light-scattering analysis of AF10 ${ }^{\mathrm{OM}-\mathrm{LZ}}$. deed, both GST pull-down and ITC studies confirmed the direct binding between the coiled-coil domains of DOT1L and AF17 (Fig. 1B; Supplemental Fig. S2B).

\section{Crystal structure of $A F 10^{O M-L Z}$}

To elucidate the molecular basis of the DOT1L-AF10 interaction, we first determined the crystal structure of AF10 ${ }^{\mathrm{OM}-\mathrm{LZ}}$ (residues 720-795) (Supplemental Table S1). The asymmetric unit contains two all-helical AF10 OM-LZ protomers in which the OMs (residues 731-750) pack against the LZs (residues 753-794) (Fig. 1D). The LZs from these two protomers dimerize and form anti-parallel coiled coils, resulting in a buried surface area of $681 \AA^{2}$ (Krissinel and Henrick 2007). Static light-scattering studies revealed that AF10 ${ }^{\mathrm{OM}-\mathrm{LZ}}$ has a measured mass of $\sim 19 \mathrm{kDa}$, which matches the theoretical mass of dimeric AF10 ${ }^{\mathrm{OM}-\mathrm{LZ}}(\sim 18 \mathrm{kDa})$ (Fig. 1E). There are 3.5 heptad repeats in each AF10 ${ }^{\mathrm{OM}-\mathrm{LZ}} \mathrm{LZ}$ domain, with predominantly hydrophobic residues at the $a$ and $d$ positions (Supplemental Fig. S3A). These hydrophobic residues are invariant across species (Supplemental Fig. S2A), suggesting that the dimerization mode is preserved among different organisms. Polar interactions are also present in the twostranded helical coiled coils, such as $\$ 762$ at the $g$ position hydrogen-bonding with Q776 at the $g^{\prime}$ position (Supplemental Fig. S3B). Substitutions of hydrophobic residues with glutamic acid significantly disrupted the homodimer formation (Supplemental Fig. S3C).

\section{Crystal structure of the DOT1L $L^{C C 2}-A F 10^{O M-L Z}$ complex}

Because the DOT1L $\mathrm{L}^{\mathrm{CC} 2}$ protein behaves better than DOT1L ${ }^{C C 1}$ and DOT1L ${ }^{C C 3}$, we chose DOT1L CC2 for structural studies of the DOT1L-AF10 interaction. To reconstitute the complex, we mixed stoichiometric amounts of recombinant human DOT1L ${ }^{\mathrm{CC} 2}$ and $\mathrm{AF} 10^{\mathrm{OM}-\mathrm{LZ}}$ proteins and monitored complex formation using analytical size exclusion chromatography. Apparent coelution of DOT1L $\mathrm{L}^{\mathrm{CC} 2}$ and AF10 ${ }^{\mathrm{OM}-\mathrm{LZ}}$ indicated formation of the DOT1L CC2 $-A F 10$ OM-LZ complex (Fig. 2A).

We next tried to crystallize the DOT1L ${ }^{\mathrm{CC}}-\mathrm{AF} 10^{\mathrm{OM}-\mathrm{LZ}}$ complex. However, we could obtain only needle-shaped crystals that diffracted poorly. Extensive optimization (including seeding, protein truncations, mutations, and DOT1L-AF10 fusion protein) of these crystals was not successful. Nevertheless, we were able to crystallize and determine the structure of a chimeric complex of human AF10 ${ }^{\mathrm{OM}-\mathrm{LZ}}$ and Danio rerio (zebrafish) DOT1L ${ }^{\mathrm{CC} 2}$ (Supplemental Table S1). The human DOT1L and zebrafish DOT1L have a high degree of sequence homology in the coiled-coil domains (Supplemental Fig. S1A), and our ITC results showed that the AF10 ${ }^{\mathrm{OM}-\mathrm{LZ}}$ binds to zebrafish DOT1L $\mathrm{L}^{\mathrm{CC} 2}$ and human DOT1L ${ }^{\mathrm{CC} 2}$ with similar affinities (Fig. 1C; Supplemental Fig. S4A). Therefore, the interactions between DOT1L $\mathrm{LC}^{\mathrm{CC}}$ and AF10 ${ }^{\mathrm{OM}-\mathrm{LZ}}$ are highly conserved, and the structural insight from the chimeric complex could be applied to the human DOT1L-AF10 interactions as well. The DOT1L ${ }^{\mathrm{CC} 2}-\mathrm{AF} 10^{\mathrm{OM}-\mathrm{LZ}}$ complex was found to form a heterotetramer in solution (Supplemental Fig. S4B). In the complex structure, each asymmetric unit contains four molecules composed of two DOT1L $\mathrm{CC}^{\mathrm{C}}-\mathrm{AF} 10^{\mathrm{OM}-\mathrm{LZ}}$ heterodimers (Fig. 2B). The heterotetramer complex structure adopts a V-shaped architecture. The two heterodimers aligned well with each other 

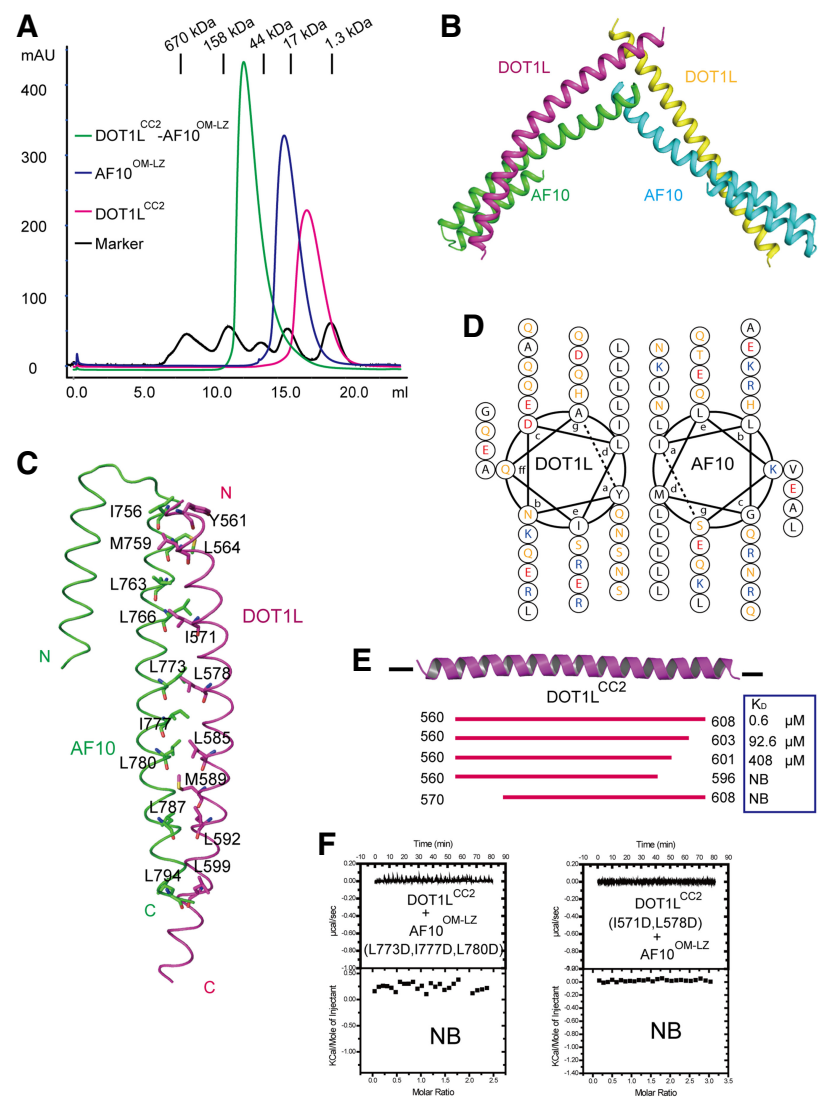

Figure 2. Structure of the DOT1L $\mathrm{L}^{\mathrm{CC} 2}-\mathrm{AF} 10^{\mathrm{OM}-\mathrm{LZ}}$ complex. (A) Superimposed gel filtration profiles of DOT1 $\mathrm{L}^{\mathrm{CC} 2}, \mathrm{AF} 10^{\mathrm{OM}-\mathrm{LZ}}$, and the DOT1L ${ }^{\mathrm{CC} 2}-\mathrm{AF} 10^{\mathrm{OM}-\mathrm{LZ}}$ complex. The elution positions of standard marker proteins are indicated at the top. The results indicate that DOT1L ${ }^{\mathrm{CC} 2}$ and AF10 $10^{\mathrm{OM}-\mathrm{LZ}}$ exist as a dimer in solution. $(B)$ Overall structure of DOT1L ${ }^{\mathrm{CC} 2}-\mathrm{AF} 10^{\mathrm{OM}-\mathrm{LZ}} \cdot(C)$ The interaction interface between DOT1L $\mathrm{L}^{\mathrm{CC} 2}$ and AF10 ${ }^{\mathrm{OM}-\mathrm{LZ}}$. DOT1L $\mathrm{L}^{\mathrm{CC} 2}$ forms the parallel coiled coils with AF10 ${ }^{\mathrm{AM}-\mathrm{LZ}}$. Hydrophobic residues at the heterodimer interface are indicated. $(D)$ Helical wheel presentation of the coiled-coil motifs formed by DOT1L $\mathrm{L}^{\mathrm{CC} 2}$ and AF10 ${ }^{\mathrm{OM}-\mathrm{LZ}}$. (E) ITCbased measurements of the AF10 ${ }^{\mathrm{OM}-\mathrm{LZ}}$ binding for wide-type and variant DOT1L ${ }^{\mathrm{CC} 2}$. $(F)$ ITC results showing that the double mutant on DOT1L or triple mutant on AF10 disrupts the complex binding.

(Supplemental Fig. S4C) and were linked by a disulfide bridge involving C604 in DOT1L ${ }^{\mathrm{CC} 2}$ (Supplemental Fig. S4D). Of note, the disulfide bond is almost absent in the larger DOT1L fragment (Supplemental Fig. S4E), thus suggesting that the observed disulfide bond may be an artifact.

Interaction of DOT1L ${ }^{C C 2}$ with $A F 10^{O M-L Z}$

The DOT1L ${ }^{\mathrm{CC} 2}-\mathrm{AF} 10^{\mathrm{OM}-\mathrm{LZ}}$ complex forms a parallel coiled-coil heterodimer (Fig. 2C), packing in a "knobs into holes" manner (Lupas 1996). Residues at the $d$ positions of both DOT1L ${ }^{\mathrm{CC} 2}$ and AF10 OM-LZ heptad repeats are all hydrophobic and highly conserved (Fig. 2D; Supplemental Figs. S1A, 2A), and hydrophobic interactions are predominantly responsible for stabilizing the heterodimer. Polar residues are prevalent at $a$ positions of both molecules, especially for the DOT1L ${ }^{\mathrm{CC} 2}$ heptad repeats. The OM of AF10 is not directly involved in the DOT1L ${ }^{\mathrm{CC} 2}$ binding, and deletion of this OM had no obvious effect on the DOT1L $\mathrm{L}^{\mathrm{CC} 2}$ binding (Supplemental Fig. S5A). However, previous studies have shown that both the OM and LZ are essential for DOT1L-AF10 interactions in vivo (DiMartino et al. 2002). Indeed, removal of the GST tag led to precipitation of virtually all of the AF10 protein without the OM, suggesting that the OM may play a role in stabilization of the LZ moiety. Similarly, deletion of the $\mathrm{N}$ terminus or $\mathrm{C}$ terminus of DOT1L $\mathrm{LC}^{\mathrm{C} 2}$ also impaired the interaction between DOT $1 \mathrm{~L}^{\mathrm{CC} 2}$ and AF10 ${ }^{\mathrm{OM}-\mathrm{LZ}}$ in vitro (Fig. 2E). To discern the residues critical for the DOT1L ${ }^{\mathrm{CC} 2}-\mathrm{AF} 10^{\mathrm{OM}-\mathrm{LZ}}$ complex formation, we introduced mutations into the $a$ and/or $d$ positions of the heptad repeats of DOT1L ${ }^{\mathrm{CC} 2}$ or AF10 ${ }^{\mathrm{OM}-\mathrm{L} Z}$. No detectable AF10 binding was observed for the double mutation (I571D/L578D) of DOT1L CC2 in either GST pull-down or ITC studies (Fig. 2F; Supplemental Fig. S5A). Likewise, the triple mutation (L773D/I777D/L780D) in AF10 OM-LZ abolished binding of the DOT1L coiled-coil domains (Figs. 1B, 2F; Supplemental Fig. S5B,C), indicating similar AF10-binding modes for these three coiled-coil domains of DOT1L. Furthermore, the majority of these key residues for AF10-binding is conserved among DOT1L ${ }^{\mathrm{CC} 1}$, DOT1L ${ }^{\mathrm{CC} 2}$, and DOT1L ${ }^{\mathrm{CC} 3}$ (Supplemental Fig. S1B).

It was reported previously that the L736R mutation in the OM or the L787R mutation in the LZ of AF10 significantly reduces its binding to DOT1L (Okada et al. 2005). Based on our complex structure, the impaired binding may arise from electrostatic repulsion between the arginine substitutions and their neighboring basic residues from AF10 or DOT1L (Supplemental Fig. S5D).

DOT1L-AF10 interaction is essential for MLL-AF10associated leukemogenesis

To evaluate the role of the DOT1L-AF10 interaction in MLL-AF10 leukemogenesis, we cotransduced DOT1Lfloxed (DOT1L $\mathrm{f}^{\mathrm{f}}$ ErCre+/-) murine bone marrow cells with pMSCB-DOT1L or pMSCB-DOT1L (I571D/L578D) together with pMSCV-MLL-AF10 or pMSCV-MLL-AF10 (L773D/I777D/L780D) (Okada et al. 2005). The effects of DOT1L or AF10 mutations on proliferation of MLL-AF10 cells were analyzed by liquid culture proliferation and colony-forming assays (Zhou et al. 2016). Expression of the DOT1L $L^{1571 D / L 578 D}$ mutation led to a significant growth inhibition of MLL-AF10 transformed cells (Fig. 3A Supplemental Fig. S6A). The triple AF10 $1773 \mathrm{D} / \mathrm{I} 747 \mathrm{D} / \mathrm{L} / 80 \mathrm{D}$ mutation almost inhibited cell proliferation and colony formation (Fig. 3A,B). Furthermore, wild-type transformed cells formed compact and blast-like colonies in the methylcellulose medium, whereas the DOT1L ${ }^{\mathrm{I571D/L} 578 \mathrm{D}}$ or $\mathrm{AF} 10^{\mathrm{L} 773 \mathrm{D} / \mathrm{I} 777 \mathrm{D} / \mathrm{L} 780 \mathrm{D}}$ mutant grew in small and diffuse colonies (Fig. 3C; Supplemental Fig. S6B). Consistent with these results, Wright-Giemsa staining suggested that DOT1L $\mathrm{L}^{\mathrm{I} 71 \mathrm{D} / \mathrm{L} 578 \mathrm{D}}$ or $\mathrm{AF} 10^{\mathrm{L} 773 \mathrm{D} / \mathrm{I} 777 \mathrm{D} / \mathrm{L} 780 \mathrm{D}}$ mutant cells display an increased myeloid differentiation with a larger cytoplasm and smaller nucleus compared with wild type (Fig. 3C; Supplemental Fig. S6B).

We next examined the roles of the DOT1L-AF10 interaction in the expression of MLL-AF10 target genes using quantitative RT-PCR. As expected, the HOXA gene cluster was significantly down-regulated in cells expressing the DOT1L $\mathrm{L}^{\mathrm{I} 71 \mathrm{D} / \mathrm{L} 578 \mathrm{D}}$ or $\mathrm{AF} 10^{\mathrm{L} 773 \mathrm{D} / \mathrm{I} 777 \mathrm{D} / \mathrm{L} 780 \mathrm{D}}$ mutant (Fig. 3D; Supplemental Fig. S6C), thereby suggesting that the expression of HOXA genes depends on DOT1L-AF10 binding. Consistently, chromatin 

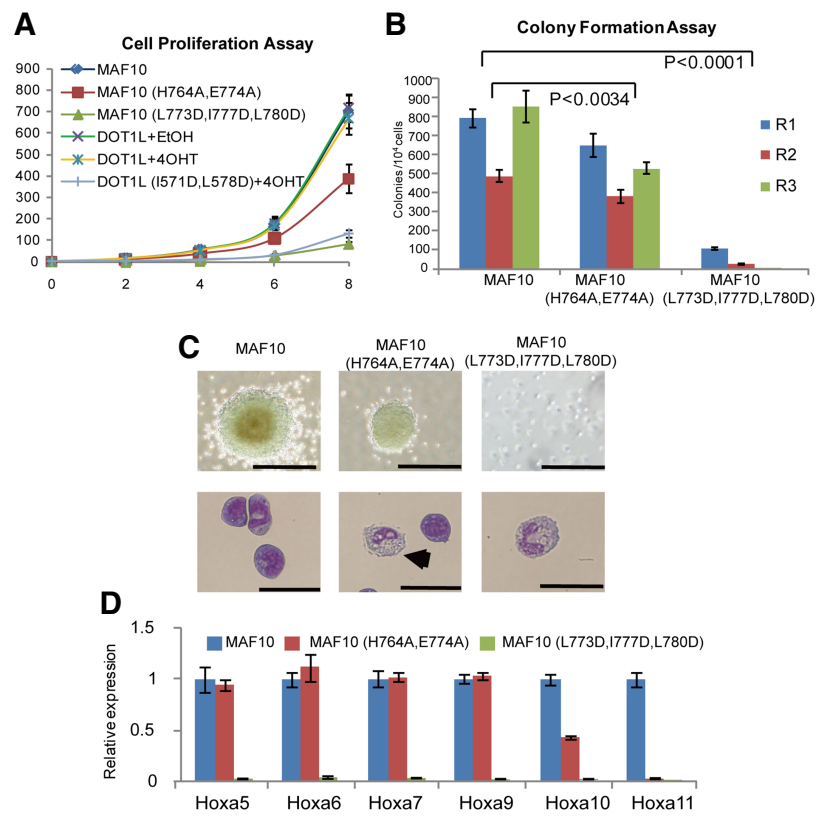

Figure 3. The DOT1L-AF10 binding is essential for MLL-AF10-associated leukemogenesis. (A) Cell proliferation assay for MLL-AF10 (MAF10) wild-type or mutant transduced bone marrow cells as well as MAF10 wild type transduced with or without DOT1L deletion bone marrow cells with DOT1L wild-type or mutant overexpression. Error bars indicate standard deviation (SD) from duplicates. The results were repeated at least three times. $(B)$ Myeloid colony formation assay for transduced bone marrow cells with MAF10 wild type or mutants as indicated. Colony counts were summarized from primary, secondary, and tertiary plating on methycellulose medium in the presence of IL3, IL6, stem cell factor (SCF), and granulocyte-macrophage colony-stimulating factor (GM-CSF). Error bars indicate SD from duplicates. The results were repeated at least three times. $P<$ 0.0001 , two-way ANOVA test. $(C)$ Representative colonies $($ top $)$ and Wright-Giemas-stained cells (bottom) from the tertiary plating of MAF10 wild type or mutant transduced bone marrow cells are shown. Bars: colonies, $200 \mu \mathrm{m}$; Wright-Giemas-stained cells, $50 \mu \mathrm{m}$. (D) Realtime PCR for HOXA genes in cells as indicated. Gene expression was normalized against GAPDH and is presented as fold change against their respective levels in MAF10 wild-type cells, which was arbitrarily set at 1 . Means and SDs (as error bars) from at least three independent experiments are presented.

immunoprecipitation studies demonstrated that the DOT1L $\mathrm{L}^{\mathrm{I571D/L} 578 \mathrm{D}}$ mutant shows a reduction in expression of HOXA genes in H3K79me2 (Supplemental Fig. S6D). Taken together, these data clearly demonstrated that the DOT1L-AF10 interactions are essential for MLL-AF10-induced oncogenic transformation.

\section{The DOT1L ${ }^{C C 2}-A F 10^{O M-L Z}$ complex specifically binds zinc}

Merging statistics for the diffraction data collected near the selenium absorption edge indicated an unexpected presence of a significant anomalous signal, which we then exploited for structure solution by means of the single-wavelength anomalous diffraction technique (Wang 1985). The sites of the most significant scatterers appeared to be coordinated by protein side chains and solvent molecules and were tentatively assigned as Zn(II) ions because a fluorescence spectrum revealed peaks that approximately corresponded to the characteristic zinc emission energies. A binding survey of various divalent cations confirmed that the DOT1L ${ }^{\mathrm{CC}}-\mathrm{AF} 10^{\mathrm{OM}-\mathrm{LZ}}$ complex specifically bound to $\mathrm{Zn}(\mathrm{II})$, with very weak or no binding detected with other divalent cations tested (Fig. 4A).

We modeled several zinc ions in the DOT1L ${ }^{\mathrm{CC}}{ }_{-}$ AF10 ${ }^{\mathrm{OM}-\mathrm{LZ}}$ heterodimer structures (Supplemental Fig. 7); two of those sites are conserved in both heterodimers (Fig. 4B). One of the $\mathrm{Zn}$ (II) ions is coordinated by Glu737 and His764 of AF10, and a second $\mathrm{Zn}(\mathrm{II})$ ion is located at an DOT1L-AF10 interface coordination site formed by Glu774 of AF10 and His574 of DOT1L (Fig. 4B), suggesting that the DOT1L-AF10 complex formation is crucial for $\mathrm{Zn}$ (II) binding. Indeed, ITC studies revealed Zn(II) dissociation constants $\left(K_{\mathrm{D}}\right)$ of $\sim 40 \mu \mathrm{M}$ for the DOT1L $\mathrm{CC}_{-}$ AF10 ${ }^{\mathrm{MM}-\mathrm{LZ}}$ complex, $\sim 600 \mu \mathrm{M}$ for AF10 ${ }^{\mathrm{MM}-\mathrm{LZ}}$, and no binding for DOT1L ${ }^{\mathrm{C} 2}$ (Fig. 4C). The positioning of $\mathrm{Zn}$ (II) ions also suggests a structural role that they may play in strengthening the DOT1L $\mathrm{L}^{\mathrm{CC}}-\mathrm{AF} 10^{\mathrm{OM}-\mathrm{LZ}}$ interaction. Indeed, the $\mathrm{ZnCl}$-containing buffer resulted in a slightly increased binding affinity between DOT1L ${ }^{\mathrm{CC} 2}$ and AF10 $10^{\mathrm{M}-\mathrm{LZ}}$ (Fig. 4D). We also observed an increased melting temperature for the protein complex in the presence of the zinc ion $\left(\sim 65^{\circ} \mathrm{C}\right)$ compared with a control in the absence of the zinc ion $\left(\sim 56^{\circ} \mathrm{C}\right)$ (Fig. $\left.4 \mathrm{E}\right)$, indicating a pronounced stabilization effect of the zinc ion. Notably, the residues involved in zinc coordination are conserved between AF10 and AF17 proteins (Supplemental Fig.
A

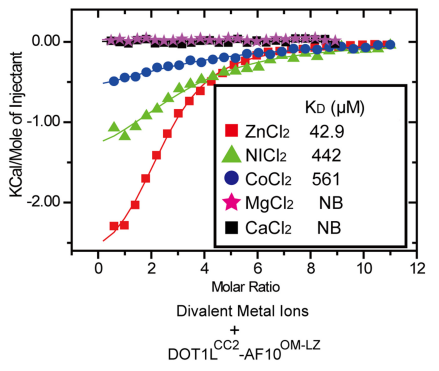

C

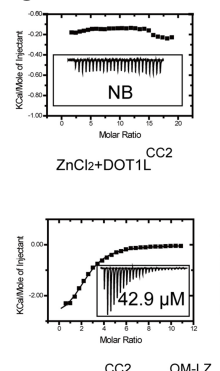

$\mathrm{ZnCl} 2+\mathrm{DOT} 1 \mathrm{~L}^{\mathrm{CC} 2}$-AF10 ${ }^{\mathrm{OM}-\mathrm{LZ}}$
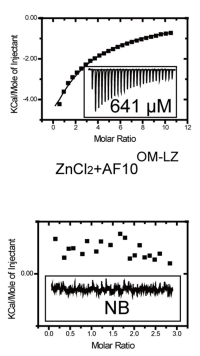

$\underset{(\mathrm{H} 764 \mathrm{~A}, \mathrm{E} 774 \mathrm{~A})}{\mathrm{ZnCl}+\mathrm{DOT})}$

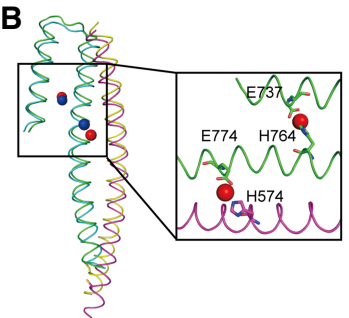

D

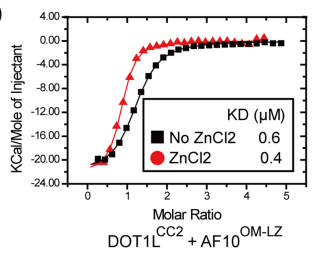

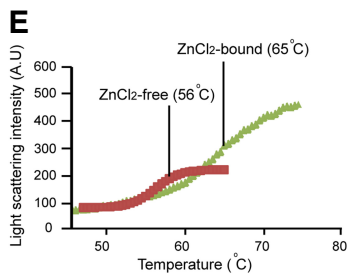

Figure 4. Zinc enhances the binding ability of DOT1L $\mathrm{L}^{\mathrm{CC} 2}$ to AF10 ${ }^{\mathrm{OM}-\mathrm{LZ}}$. (A) Summary of the quantitative binding constants between various divalent metal ions and the DOT1L ${ }^{\mathrm{CC} 2}-\mathrm{AF} 10^{\mathrm{OM}-\mathrm{LZ}}$ complex. $(B)$ There are two conserved zinc-binding sites in the DOT1L ${ }^{\mathrm{CC} 2}-\mathrm{AF} 10^{\mathrm{OM}-\mathrm{LZ}}$ complex. $(C)$ Dissociation constants of zinc with DOT1L ${ }^{\mathrm{CC} 2}, \mathrm{AF} 10^{\mathrm{OM}-\mathrm{LZ}}$, and the DOT1L ${ }^{\mathrm{CC} 2}-\mathrm{AF} 10^{\mathrm{OM}-\mathrm{LZ}} \mathrm{com}-$ plex. Mutation of zinc-coordinating residues $\left(\mathrm{AF} 10^{\mathrm{H} 764 \mathrm{~A} / \mathrm{E} 774 \mathrm{~A}}\right)$ abolishes the zinc binding. $(D)$ ITC data show that zinc enhances the binding of DOT1L $\mathrm{L}^{\mathrm{CC} 2}$ to AF10 $\mathrm{OM}-\mathrm{LZ}$. (E) The thermal shift melting curves of the DOT1L $\mathrm{L}^{\mathrm{CC} 2}-\mathrm{AF} 10^{\mathrm{OM}-\mathrm{LZ}}$ complex in the presence or absence of zinc measured by differential static light scattering. 
S2A), implying that they have a conserved function in zinc binding. Indeed, the zinc ion exhibited a similar effect on the DOT1L ${ }^{\mathrm{CC} 2}-\mathrm{AF} 17^{\mathrm{OM}-\mathrm{LZ}}$ complex, suggesting a conserved role of $\mathrm{Zn}(\mathrm{II})$ in MLL-AF10/AF17-associated leukemogenesis (Supplemental Fig. S2C).

\section{A role of zinc in the leukemogenesis}

Zinc is involved in numerous cellular pathways and is essential for normal functions. Abnormal cellular zinc homeostasis has been associated with many diseases, including cancer, diabetes, and neurodegeneration (Maret 2013). Previous studies suggest that zinc functions as a regulator of monocyte differentiation in the acute myeloid leukemia cell line HL-60 (Dubben et al. 2010). To test the validity of the $\mathrm{Zn}$ (II) binding observed in the crystal structure, we mutated residues involved in zinc coordination and then assessed leukemogenesis. Double mutation (H764A/E774A) of AF10 abolished its zinc-binding ability as examined by ITC (Fig. 4C). Consistent with the modest role of zinc in mediating the DOT1L-AF10 binding, $\mathrm{AF} 10^{\mathrm{H} 764 \mathrm{~A} / \mathrm{E} 774 \mathrm{~A}}$ had a modest inhibitory effect on proliferation and differentiation of MLL-AF10 cells (Fig. 3AC). On the other hand, although the expression of Hoxa7 and $H$ Hoxa9 was just slightly affected in cells expressing the H764A/E774A mutant, Hoxa10 and Hoxa11 were significantly down-regulated. Furthermore, a decrease of H3K79me2 at the Hoxa10 and Hoxa11 was detected (Supplemental Fig. S6E). Given that overexpression of Hoxa10 results in suppression of megakaryocyte (MK) development (Magnusson et al. 2007), zinc may play a role in MLL-AF10-mediated leukemogenesis through the regulation of postnatal hematopoietic development. Taken together, zinc ions may promote leukemogenesis by supporting cell proliferation and Hoxa10/Hoxa11 expression via enhancing the DOT1L-AF10 interactions.

In summary, we characterized the DOT1L-AF10 interaction by providing the first structural model for how AF10 recruits DOT1L and probed the effect of this interaction on MLL-AF10-mediated leukemic transformation. Apart from binding of AF10 and AF17, DOT1L is also involved in the binding of other MLL fusion partners such as AF9 and ENL (Zhang et al. 2006; Mueller et al. 2007; Kuntimaddi et al. 2015), albeit through distinct binding sites: AF10 and AF17 specifically interact with the coiled-coil domains of DOT1L based on our results, whereas AF9 and ENL bind mainly to the C-terminal intrinsically disordered region of DOT1L (Mueller et al. 2007; Kuntimaddi et al. 2015). Although AF9 and AF10 bind to DOT1L through different binding sites, both binding events involve more than one binding site on DOT1L. The three DOT1L-binding sites for AF10 are proximal to each other (Fig. 1A); in contrast, AF9 binds to three spatially separated sites of DOT1L (Kuntimaddi et al. 2015). Multiple binding motifs on DOT1L would increase the DOT1L-binding affinity and enhance HOXA gene expression, an event reminiscent of the role of oligomerization proposed for MLL. Oligomerization of MLL via the self-association motifs of fusion partners has been reported to be important and sufficient to immortalize hematopoietic cells (Martin et al. 2003; Smith et al. 2017). Our DOT1LAF10 data together with the previous DOT1L-AF9 studies revealed another mode of oligomerization of the MLL fusion proteins; i.e., heterodimerization between DOT1L and AF9 or AF10.
Interestingly, the first DOT1L-binding site for AF9, which spans residues 628-653, partially overlaps with the DOT1L-binding site for AF10. Consistently, AF17 has been reported to compete with AF9 for interaction with DOT1L, thereby regulating the DOT1L expression (Reisenauer et al. 2009). Thus, it is conceivable that AF10 would also compete with AF9 for interaction with DOT1L.

The DOT1L-AF10 interaction has been implicated in regulating the HOXA gene cluster expression in various AML subtypes (Deshpande et al. 2014), unveiling that the DOT1L-AF10-binding interface would be an excellent therapeutic target for AML. In addition, blocking the interaction of MLL-AF10 with DOT1L is likely to have some advantages over inhibiting the DOT1L enzymatic activity, as the MLL-AF10 targeted therapy would be of high efficacy and low toxicity. Recently, it was shown that disruption of GAS41-AF10 ${ }^{\mathrm{OM}-\mathrm{LZ}}$ binding by the inhibitory peptides results in a significant reduction in HOXA gene cluster expression, further suggesting that blocking the DOT1L-AF10 interaction may be a feasible therapeutic approach in treating leukemias (Hagen et al. 2014). Similarly, the peptide mimics and small molecules that target the coiled-coil domain of HIV-1 gp41 have been successfully developed and applied to the HIV therapy (Liu et al. 2007; Welch et al. 2007; Sofiyev et al. 2017). Thus, our structures presented here could serve as a foundation for the development of specific DOT1LAF10 ${ }^{\mathrm{M}-\mathrm{LZ}}$ targeted drugs in the future.

\section{Materials and methods}

\section{Protein crystallization}

Crystallization experiments were performed using sitting drop vapor diffusion methods at $18^{\circ} \mathrm{C}$. Crystals of AF10 ${ }^{\mathrm{OM}-\mathrm{LZ}}$ were grown by mixing $1 \mu \mathrm{L}$ of protein solution with $1 \mu \mathrm{L}$ of reservoir solution consisting of $0.2 \mathrm{M}$ magnesium pernitrate $(\mathrm{pH} 5.5)$ and $20 \%(\mathrm{w} / \mathrm{v})$ PEG3350. The DOT1L ${ }^{\mathrm{CC}}$ AF10 ${ }^{\text {OM-LZ }}$ crystals were obtained in the mixture solution of $0.1 \mathrm{M}$ sodium acetate ( $\mathrm{pH} 4.6), 0.2 \mathrm{M}$ calcium chloride, $0.01 \mathrm{M}$ zinc chloride, and $30 \%(\mathrm{w} /$ v) MPD. Crystals appeared in $3 \mathrm{~d}$ and grew to full size in $3 \mathrm{wk}$. Before data collection, the crystals were soaked in the reservoir solution supplemented with $10 \%$ glycerol before being flash-frozen.

Additional experimental details are in the Supplemental Material.

\section{Acknowledgments}

We thank Dr. Akihiko Yokoyama for providing the pMSCV-MLL-AF10 plasmids, Dr. Guillermo Senisterra for help with the differential static light scattering, and the Ontario Institute for Cancer Research for access to multiangle light-scattering equipment. We also thank Dr. Rui-ming $\mathrm{Xu}$ for communicating their data prior to publication. Some X-ray diffraction experiments were performed at Argonne National Laboratory Structural Biology Center (SBC-CAT) Beamline 19-ID at the Advanced Photon Source. SBC-CAT is operated by University of Chicago Argonne, LLC, for the U.S. Department of Energy, Office of Biological and Environmental Research, under contract DE-AC02-06CH11357. Beamline 08ID-1 at the Canadian Light Source is supported by the Canada Foundation for Innovation, Natural Sciences, and Engineering Research Council of Canada; the University of Saskatchewan; the Government of Saskatchewan; Western Economic Diversification Canada; the National Research Council Cana$\mathrm{da}$; and the Canadian Institutes of Health Research. The Structural Genomics Consortium is a registered charity (no. 1097737) that receives funds from AbbVie; Bayer Pharma AG; Boehringer Ingelheim; Canada Foundation for Innovation; Eshelman Institute for Innovation; Genome Canada through Ontario Genomics Institute (OGI-055); Innovative Medicines Initiative (European Union/European Federation of Pharmaceutical Industries and Associations [EU/EFPIA]; Unrestricted Leveraging of Targets 
for Research Advancement and Drug Discovery [ULTRA-DD] grant no. 115766); Janssen; Merck KGaA; Merck Sharp and Dohme; Novartis Pharma AG; Ontario Ministry of Research, Innovation, and Science (MRIS); Pfizer; São Paulo Research Foundation (FAPESP); Takeda; and Wellcome. This work is also supported by the Natural Sciences and Engineering Research Council of Canada (NSERC; funding reference no. RGPIN-201606300).

Author contributions: H.Z., Y.D., and J.M. designed the research; H.Z., B.Z., S.Q., J.X., R.H., W.T., V.N., Y.L., and P.L. performed the research; H.Z., Y.D., and J.M. analyzed the data; and H.Z. and J.M. wrote the paper.

\section{References}

Ayton PM, Cleary ML. 2003. Transformation of myeloid progenitors by MLL oncoproteins is dependent on Hoxa7 and Hoxa9. Genes Dev 17: 2298-2307.

Benjamin DY, Hess JL, Horning SE, Brown GA, Korsmeyer SJ. 1995. Altered Hox expression and segmental identity in Mll-mutant mice. $\mathrm{Na}$ ture 378: 505-508.

Chen L, Deshpande AJ, Banka D, Bernt KM, Dias S, Buske C, Olhava EJ, Daigle SR, Richon VM, Pollock RM. 2013. Abrogation of MLL-AF10 and CALM-AF10-mediated transformation through genetic inactivation or pharmacological inhibition of the H3K79 methyltransferase Dot11. Leukemia 27: 813-822.

Cosgrove MS, Patel A. 2010. Mixed lineage leukemia: a structure-function perspective of the MLL1 protein. FEBS J 277: 1832-1842.

Deshpande AJ, Deshpande A, Sinha AU, Chen L, Chang J, Cihan A, Fazio M, Chen C-w, Zhu N, Koche R. 2014. AF10 regulates progressive H3K79 methylation and HOX gene expression in diverse AML subtypes. Cancer Cell 26: 896-908.

DiMartino JF, Ayton PM, Chen EH, Naftzger CC, Young BD, Cleary ML. 2002. The AF10 leucine zipper is required for leukemic transformation of myeloid progenitors by MLL-AF10. Blood 99: 3780-3785.

Dubben S, Hönscheid A, Winkler K, Rink L, Haase H. 2010. Cellular zinc homeostasis is a regulator in monocyte differentiation of HL-60 cells by 1a, 25-dihydroxyvitamin D3. J Leukoc Biol 87: 833-844.

Finn RD, Clements J, Eddy SR. 2011. HMMER Web server: interactive sequence similarity searching. Nucleic Acids Res 39: W29-W37.

Hagen S, Mattay D, Räuber C, Müller KM, Arndt KM. 2014. Characterization and inhibition of AF10-mediated interaction. I Pept Sci 20: 385-397.

Krissinel E, Henrick K. 2007. Inference of macromolecular assemblies from crystalline state. I Mol Biol 372: 774-797.

Krivtsov AV, Armstrong SA. 2007. MLL translocations, histone modifications and leukaemia stem-cell development. Nat Rev Cancer 7: 823-833.

Kuntimaddi A, Achille NJ, Thorpe J, Lokken AA, Singh R, Hemenway CS, Adli M, Zeleznik-Le NJ, Bushweller JH. 2015. Degree of recruitment of DOT1L to MLL-AF9 defines level of H3K79 di- and tri-methylation on target genes and transformation potential. Cell Rep 11: 808-820.

Liu S, Wu S, Jiang S. 2007. HIV entry inhibitors targeting gp41: from polypeptides to small-molecule compounds. Curr Pharm Des 13: 143-162.

Lupas A. 1996. Coiled coils: new structures and new functions. Trends Biochem Sci 21: 375-382.

Magnusson M, Brun AC, Miyake N, Larsson J, Ehinger M, Bjornsson JM, Wutz A, Sigvardsson M, Karlsson S. 2007. HOXA10 is a critical regula- tor for hematopoietic stem cells and erythroid/megakaryocyte development. Blood 109: 3687-3696.

Maret W. 2013. Zinc and human disease. Met Ions Life Sci 13: 389-414.

Martin ME, Milne TA, Bloyer S, Galoian K, Shen W, Gibbs D, Brock HW, Slany R, Hess JL. 2003. Dimerization of MLL fusion proteins immortalizes hematopoietic cells. Cancer Cell 4: 197-207.

Meyer C, Schneider B, Jakob S, Strehl S, Attarbaschi A, Schnittger S, Schoch C, Jansen M, van Dongen J, Den Boer M. 2006. The MLL recombinome of acute leukemias. Leukemia 20: 777-784.

Mueller D, Bach C, Zeisig D, Garcia-Cuellar M-P, Monroe S, Sreekumar A, Zhou R, Nesvizhskii A, Chinnaiyan A, Hess JL. 2007. A role for the MLL fusion partner ENL in transcriptional elongation and chromatin modification. Blood 110: 4445-4454.

Muntean AG, Hess JL. 2012. The pathogenesis of mixed-lineage leukemia. Annu Rev Pathol 7: 283-301.

Okada Y, Feng Q, Lin Y, Jiang Q, Li Y, Coffield VM, Su L, Xu G, Zhang Y. 2005. hDOT1L links histone methylation to leukemogenesis. Cell 121: $167-178$.

Okada Y, Jiang Q, Lemieux M, Jeannotte L, Su L, Zhang Y. 2006. Leukaemic transformation by CALM-AF10 involves upregulation of Hoxa5 by hDOT1L. Nat Cell Biol 8: 1017-1024.

Reisenauer MR, Anderson M, Huang L, Zhang Z, Zhou Q, Kone BC, Morris AP, LeSage GD, Dryer SE, Zhang W. 2009. AF17 competes with AF9 for binding to Dotla to up-regulate transcription of epithelial $\mathrm{Na}^{+}$ channel a. J Biol Chem 284: 35659-35669.

Shilatifard A. 2012. The COMPASS family of histone H3K4 methylases: mechanisms of regulation in development and disease pathogenesis. Annu Rev Biochem 81: 65-95.

Slany R. 2016. The molecular mechanics of mixed lineage leukemia. Oncogene 35: 5215-5223.

Smith MJ, Ottoni E, Ishiyama N, Goudreault M, Haman A, Meyer C, Tucholska M, Gasmi-Seabrook G, Menezes S, Laister RC. 2017. Evolution of AF6-RAS association and its implications in mixed-lineage leukemia. Nat Commun 8: 1099.

Sofiyev V, Kaur H, Snyder BA, Hogan PA, Ptak RG, Hwang P, Gochin M. 2017. Enhanced potency of bivalent small molecule gp41 inhibitors. Bioorg Med Chem 25: 408-420.

Thirman MJ. 2017. Paradoxical effects of MLL paralogs in MLL-rearranged leukemia. Cancer Cell 31: 729-731.

Wang B-C. 1985. Resolution of phase ambiguity in macromolecular crystallography. Methods Enzymol 115: 90-112.

Welch BD, VanDemark AP, Heroux A, Hill CP, Kay MS. 2007. Potent Dpeptide inhibitors of HIV-1 entry. Proc Natl Acad Sci 104: 16828-16833.

Yoshida T, Ng SY-M, Zuniga-Pflucker JC, Georgopoulos K. 2006. Early hematopoietic lineage restrictions directed by Ikaros. Nat Immunol 7: 382-391.

Zhang W, Xia X, Reisenauer MR, Hemenway CS, Kone BC. 2006. DotlaAF9 complex mediates histone H3 Lys-79 hypermethylation and repression of $\mathrm{ENaCa}$ in an aldosterone-sensitive manner. I Biol Chem 281: 18059-18068.

Zhou B, Wang J, Lee SY, Xiong J, Bhanu N, Guo Q, Ma P, Sun Y, Rao RC, Garcia BA. 2016. PRDM16 suppresses MLL1r leukemia via intrinsic histone methyltransferase activity. Mol Cell 62: 222-236.

Ziemin-van der Poel S, McCabe NR, Gill HJ, Espinosa R, Patel Y, Harden A, Rubinelli P, Smith SD, LeBeau MM, Rowley JD. 1991. Identification of a gene, MLL, that spans the breakpoint in 11q23 translocations associated with human leukemias. Proc Natl Acad Sci 88: 10735-10739. 


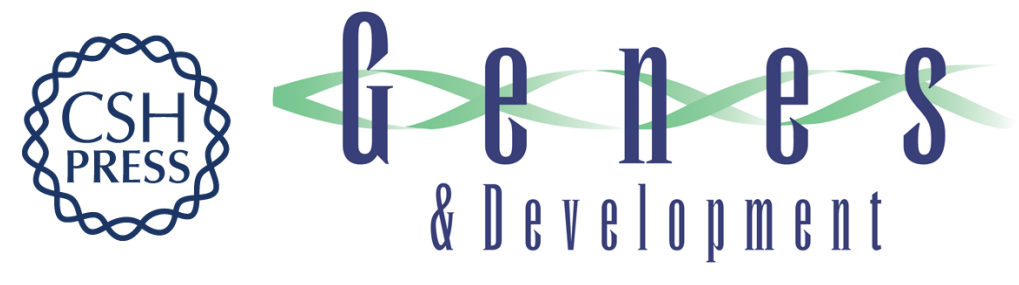

\section{Structural and functional analysis of the DOT1L-AF10 complex reveals mechanistic insights into MLL-AF10-associated leukemogenesis}

Heng Zhang, Bo Zhou, Su Qin, et al.

Genes Dev. 2018, 32: originally published online March 21, 2018

Access the most recent version at doi:10.1101/gad.311639.118

Supplemental Material

References

Creative

Commons

License

Email Alerting

Service
http://genesdev.cshlp.org/content/suppl/2018/03/21/gad.311639.118.DC1

This article cites 34 articles, 8 of which can be accessed free at: http://genesdev.cshlp.org/content/32/5-6/341.full.html\#ref-list-1

This article is distributed exclusively by Cold Spring Harbor Laboratory Press for the first six months after the full-issue publication date (see http://genesdev.cshlp.org/site/misc/terms.xhtml). After six months, it is available under a Creative Commons License (Attribution-NonCommercial 4.0 International), as described at http://creativecommons.org/licenses/by-nc/4.0/.

Receive free email alerts when new articles cite this article - sign up in the box at the top right corner of the article or click here.

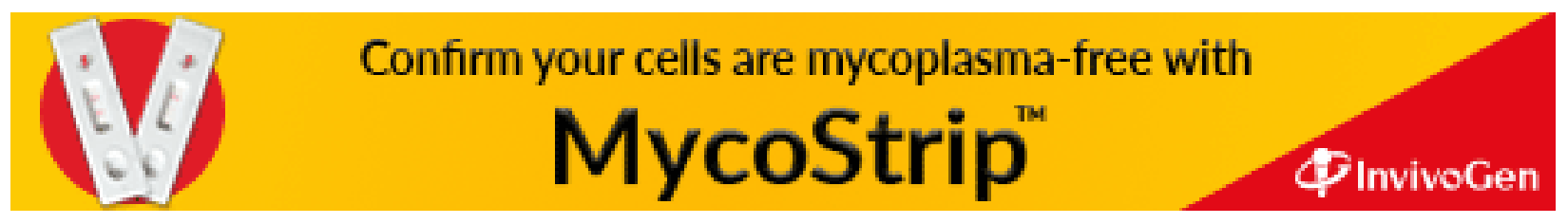

